\title{
AN EXPERIMENTAL STUDY OF VORTEX-FLAME INTERACTION IN COUNTERFLOW SPRAY DIFFUSION FLAMES
}

\author{
VITO S. SANTORO, DIMITRIOS C. KYRITSIS AND ALESSANDRO GOMEZ \\ Yale Center for Combustion Studies \\ Yale University \\ New Haven, CT 06520-7682, USA
}

\begin{abstract}
The extinction behavior of methanol counterflow spray diffusion flames was investigated using a combination of formaldehyde planar laser-induced fluorescence (PLIF) and phase Doppler measurements. Extinction was brought about quasi-steadily, by progressively increasing the flow rates of both oxidizer and fuel side, and unsteadily, by generating a vortex on the oxidizer side. The unsteady experiments yielded values of extinction strain rates a factor of 2 larger than the quasi-steady values. The greater robustness of the spray flame under unsteady perturbation was explained phenomenologically by estimating the timescales involved in the process. It was found that the vortex introduces unsteady effects in the outer diffusiveconvective layer of the flame. The inner reactive-diffusive layer, on the other hand, behaves in a quasisteady manner, since the characteristic chemical time is much smaller than the characteristic unsteady time. As a result, even though the instantaneous strain rate is much larger than the quasi-steady extinction strain rate, the flame is subject to a damped strain rate through the outer layer. An estimate of the thickness of the mixing layer, based on formaldehyde PLIF, provided a convenient means to compare the scalar dissipation rate and the Damköhler number between the two extinction modes, bypassing the need for detailed species measurements for the assessment of the mixture fraction and its gradient. Such a comparison showed that the difference between the two extinction modes was reduced to $25 \%$ on the average, consistent with expectations based on flame structure models from asymptotic theory. Spray flames exhibited longer time delays between the onset of extinction and reignition, as compared to gaseous flames. Estimates of the relevant Stokes number suggested that the difference may be attributed to droplet inertia effects.
\end{abstract}

\section{Introduction}

The interaction of laminar counterflow spray diffusion flames with laminar vortices was investigated experimentally. The objective was to examine key features of a well-controlled phenomenon that is relevant to practical combustion systems. This approach was motivated by the consideration that practical turbulent combustion systems are not well suited for fundamental studies because of the many difficulties that hinder the design of well-defined experiments and because of limitations in current computational and diagnostic capabilities. Conversely, the use of an environment that is intermediate in complexity between steady laminar flames and fully turbulent ones offered the capability to control the system, characterize it, and interpret the results unambiguously.

Marble was the first to analyze the evolution of a planar diffusion flame in the field of a vortex [1]. At very large values of the Péclet number, that is defined as the ratio of vortex circulation to a scalar diffusion coefficient, this analysis yielded a flame wrapped around the vortex. More realistic and moderate Péclet numbers were considered in a subsequent analytical model, and it was reported that a flame tongue forms, the stoichiometric contour being only partially wrapped around the vortex [2]. Direct numerical simulation of flame-vortex interaction was modeled with one-step kinetics and compared with laminar flamelet library predictions obtained from asymptotic analysis in a subsequent insightful study [3]. A turbulent combustion spectral diagram was also presented, and limits of the laminar flamelet approach were addressed. Experimentally, the interaction of a vortex with a laminar flame was pioneered in a premixed flame environment (see, for example, Refs. $[4,5])$. For gaseous diffusion flames, the first qualitative experimental investigation was reported in Ref. [6], followed by a number of contributions on hydrogen-air flames [7-10].

Since the majority of practical non-premixed combustion originates from liquid fuels in some form of turbulent spray flames, with the presence of the droplet phase making the turbulent environment even more intractable, an investigation on the perturbation introduced by laminar vortex rings on counterflow laminar spray diffusion flames is needed for the same reasons that motivated the recent blossoming of similar studies in gaseous environments. Yet to date, only one isolated theoretical study has been reported on the subject [11]. The 


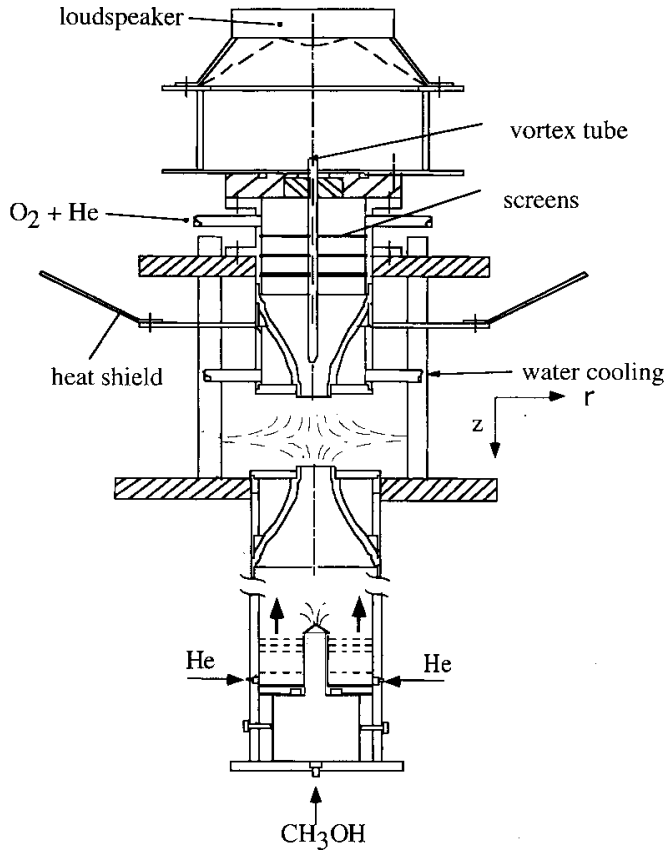

FIG. 1. Burner configuration.

present study, building on our previous work on steady spray counterflow diffusion flames $[12,13]$, addresses this issue by focusing on the quantitative characterization of the vortex-induced extinction/ reignition behavior, under conditions in which the fuel is admitted as a fine spray of droplets. Preliminary results were reported in Ref. [14].

\section{Experimental Methods}

An axisymmetric counterflow diffusion flame was established in vertical configuration, as shown in Fig. 1 , with the oxidizer being fed from one side, methanol and inert from the other. The liquid was dispersed using a commercial ultrasonic nebulizer. This device atomized modest liquid flow rates, imparting a small velocity to liquid drops with a mean diameter $d_{10}=26 \mu \mathrm{m}$ and maintaining the relative standard deviation (ratio of standard deviation over mean diameter) to values below 0.4. Both sides of the burner terminated in a contraction, contoured following a well-established wind tunnel design practice, that optimized the uniformity of the axial velocity in the radial direction. The exit diameter of the two nozzles was $12.5 \mathrm{~mm}$, and the separation distance between them was kept constant at $13 \mathrm{~mm}$. The steady flame was perturbed by periodically generated laminar toroidal vortices from the oxidizer side. A suitably synthesized voltage function was applied across the loudspeaker, causing the latter to force air through a $1.5 \mathrm{~mm}$ tube impulsively, similarly to Ref. [4]. The amplitude of the voltage function was used to control the strength of the vortex generated in this fashion.

The vortices were visualized using planar light scattering of submicron $\mathrm{TiO}_{2}$ particles, produced by hydrolysis of $\mathrm{TiCl}_{4}$. To monitor the flame dynamics under vortex excitation, formaldehyde planar laserinduced fluorescence (PLIF) was used as a complementary marker of the flame, for reasons clarified in the following section. The third harmonic of a Nd:YAG laser (355 nm and $120 \mathrm{~mJ} /$ pulse) was used to excite the tail of the $4_{0}^{1}$ transition in the $\tilde{A}^{1} A_{2} \leftarrow$ $\tilde{X}^{1} A_{1}$ band of HCHO, as in Ref. [15]. The resulting signal was detected using a gated single-stage image intensifier coupled to a charge-coupled device (Santa Barbara Instrument Group ST6B). A narrow bandpass interference filter at $415 \mathrm{~nm}$ with a full width at half-maximum of $7 \mathrm{~nm}$ was used to reject flame luminescence and other interferences. Because of the spray diluteness, droplet Mie scattering generally did not require additional filtering. Both planar diagnostic techniques were synchronized with the signal generator that produced the vortex, using a variable time delay. In this way, the evolution of the interaction between the vortex and flame could be monitored at any desired instant in time.

These diagnostic techniques were complemented by measurements of gas velocity and droplet diameter by a commercial phase Doppler anemometer. The velocity of the gas on the oxidizer side was determined using seed particles of $\mathrm{Al}_{2} \mathrm{O}_{3}$, with a nominal diameter of $1.5 \mu \mathrm{m}$. Droplets with diameter smaller than $8 \mu \mathrm{m}$ were used to evaluate the gas velocity on the fuel side. The single-point velocity measurements were phase averaged and used to evaluate the strain rate along the centerline of the flame and major vortex parameters, such as diameter, core-to-core distance, translational velocity, rotational velocity, and circulation.

In the present investigation, various flames were studied under vortex excitation. Vortex size, baseline strain rate, and fuel mass fraction were kept constant at $1.2 \mathrm{~mm}, 55 \mathrm{~s}^{-1}$, and 0.67 , respectively. The oxidizer mass fraction was varied between 0.75 and 0.90 , and helium was used as inert in both fuel and oxidizer streams.

\section{Results and Discussion}

\section{HCHO Laser-Induced Fluorescence as a Flame Marker}

The difficulty in selecting the appropriate tracer to monitor the flame behavior was emphasized in Ref. [16], where it was concluded that, at least for premixed flames, the formyl radical, HCO, is the ideal candidate since its fluorescence correlates well 


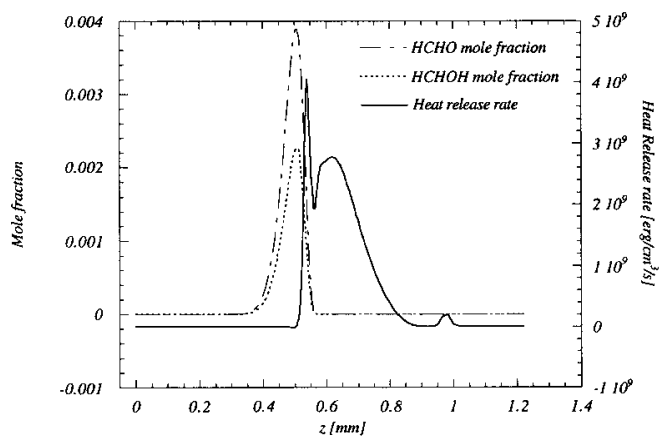

FIG. 2. Calculations of heat release rate and $\mathrm{HCHOH}$ and $\mathrm{HCHO}$ mole fraction profiles in a typical methanol spray counterflow diffusion flame under steady-state conditions.

spatially and in intensity with the peak of the heat release rate. Unfortunately, HCO PLIF is very difficult to measure. We chose instead to examine whether $\mathrm{HCHO}$, its precursor in the dominant methanol oxidation path $\left(\mathrm{CH}_{3} \mathrm{OH} \rightarrow \mathrm{HCHOH} \rightarrow\right.$ $\mathrm{HCHO} \rightarrow \mathrm{HCO} \rightarrow \mathrm{CO}$ ), could be useful for the same purpose. The pyrolysis of $\mathrm{HCHOH}$ to $\mathrm{HCHO}$ had, in fact, been suggested as the critical step for extinction in methanol flames [17]. The suitability of HCHO laser-induced fluorescence was confirmed by the results of a computational model of a steadystate counterflow diffusion flame, with detailed kinetics and transport [12], that indicated that for methanol flames the peak of heat release is juxtaposed to the location where the formaldehyde concentration precipitously drops. These numerical calculations were repeated for a range of conditions, and typical results are presented in Fig. 2, where the $\mathrm{HCHOH}$ and $\mathrm{HCHO}$ mole fractions and heat release rate are plotted as a function of the axial position. Clearly, formaldehyde is well suited as a complementary marker of the flame by virtue of this juxtaposition with the peak heat release rate position.

There is an additional bonus for using the easily accessible HCHO PLIF to probe the flame structure. Critical aspects of combustion physics can be explained by a simplified flame structure, as heralded by asymptotics studies with one-step kinetics [18]. Such a structure entails three zones: an inner zone, where convective effects are unimportant and a diffusive-reactive balance exists, and two outer zones, sandwiching the inner zone and for which a convective-diffusive balance exists. Fig. 2 clearly shows that one edge of the formaldehyde layer is adjacent to the region where the peak in heat release rate is achieved, which for the purpose of this discussion can be identified with the reactive-diffusive inner layer. The other edge of the layer, the one closer to the fuel port, can be identified as the location of the onset of fuel pyrolysis. In fact, the thickness of the HCHOH and HCHO "layers" are virtually the same, as shown in Fig. 2, and $\mathrm{HCHOH}$ is the very first intermediate in the reaction pathway. As a result, in the first approximation, the thickness of the HCHO layer can be interpreted as a measure of the thickness of the convective-diffusive (outer) layer on the fuel side, which should enable us to probe experimentally how this convective-diffusive layer responds to the imposed perturbation.

With regard to the possible interference of polycyclic aromatic hydrocarbons (PAHs) with HCHO PLIF, we note that the spatial profile of the fluorescence signal coincided with the HCHO concentration data from the computational model. Also, when flame broadband chemiluminescence and HCHO fluorescence were acquired in the same image, the fluorescence signal was separated from the chemiluminescence region by a thin dark region where no signal was recorded. The position of this region and its thickness corresponded to the numerical prediction for the HCO spatial profile, which is consistent with our attribution of the fluorescence signal to formaldehyde. If PAH had been responsible for the fluorescence, there would have been no mechanism to explain the existence of this dark zone since in the absence of soot one could not account for the PAH disappearance.

\section{Extinction Behavior}

In Fig. 3, a selected sequence of single-shot PLIF images is presented for the spray flame with $Y_{\mathrm{O}_{2}}=$ 0.9. These images show critical aspects of the interaction. The vortex, $18 \mathrm{~ms}$ after being generated, approached the flame, curving it. The interaction evolved, with the flame moving toward the fuel side, because of the increased momentum from the opposite side, and the formaldehyde layer becoming thinner, because of the momentary increase in strain rate. At $t=22 \mathrm{~ms}$, the flame was not able to sustain the high strain rate, and a hole developed. The extinction front propagated very rapidly in the radial direction, helped by the radial flow. At $t=48 \mathrm{~ms}$, after the passage of the vortex, the now annular flame closed in and re-established a flat and uniform diffusion flame, moving back to the original position.

Once the conditions at which extinction occurred were established using the PLIF images, the temporal evolution of the strain rates was measured. For the same conditions of the flame shown in Fig. 3, the time trace of the axial velocity component along the centerline was measured and phase averaged. For the sake of clarity, only selected results are plotted in Fig. 4 at a few axial locations, $1.5 \mathrm{~mm}, 4.5$ $\mathrm{mm}$, and $11.5 \mathrm{~mm}$ below the oxidizer nozzle. Clearly, the velocity profile was strongly perturbed from its 


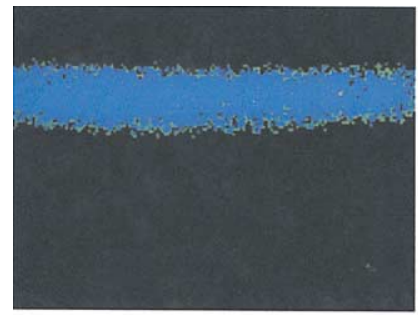

a)

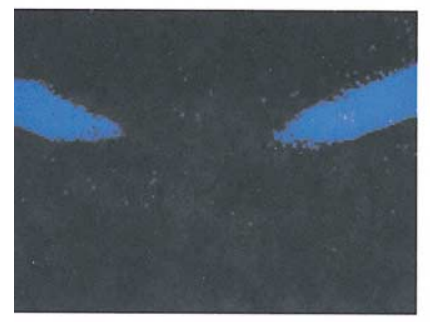

c)

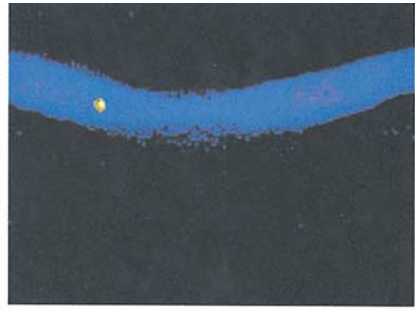

b)

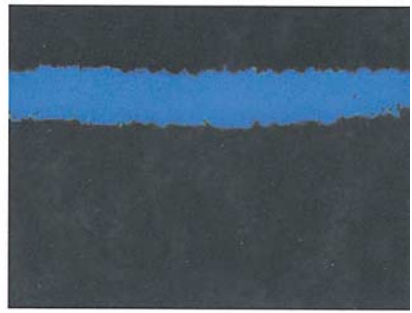

d)

$1 \mathrm{~mm}$

FIG. 3. Sequence of PLIF images during vortex-flame interaction for a prototypical spray flame. The times elapsed after vortex generation are (a) $t=0 \mathrm{~ms}$, (b) $t=20 \mathrm{~ms}$, (c) $t=22 \mathrm{~ms}$, and (d) $t=48 \mathrm{~ms}$.

steady-state value to a minimum, and then it recovered the original value. To reinterpret these results in terms of strain rate, the velocity measurements are plotted at fixed times as a function of the axial coordinate, as shown in Fig. 5, and the strain rate was calculated as the local derivative, $\partial v / \partial z$, evaluated across the five right-most data points in the figure, corresponding to the last millimeter before the flame. The strain rate increased, reached a maximum, and then relaxed back to its initial value. The extinction strain rate could be evaluated using the PLIF images together with the velocity measurements.

This procedure was repeated for various flames, keeping $Y_{\mathrm{F}}$ and the strain rate constant and reducing $Y_{\mathrm{O}_{2}}$. The results, normalized with the quasi-steady extinction strain rate, are shown in Fig. 6 as open circles. The quasi-steady extinction strain values were obtained by increasing the mass flux from fuel and oxidizer sides slowly, until extinction was observed. The vortex-induced extinction strain rates are larger than the quasi-steady values by a factor ranging between 1.8 and 2.2, indicating a greater apparent robustness of the flame to unsteady perturbations.

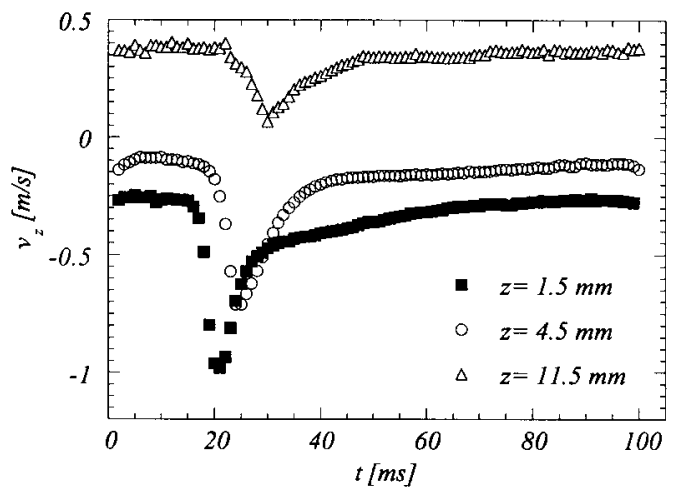

FIG. 4. Phase-averaged axial velocity measurements as a function of time at three locations along the centerline of the spray flame.

A qualitative explanation of this difference can be provided by considering first the timescales involved in the problem. Specifically, one should consider the mechanical time, $\tau_{\mathrm{m}}$, that is the inverse of the baseline strain rate, $1 / K$; the chemical time, $\tau_{\mathrm{ch}}=1 /$ $\left(K \beta^{2}\right)$, that can be evaluated from the steady-state 


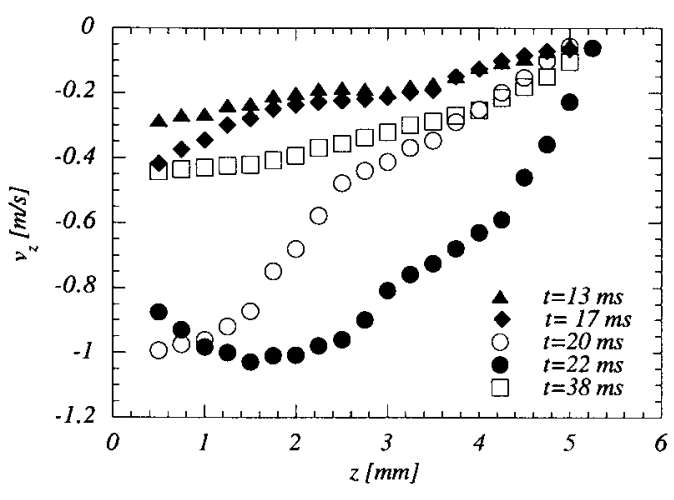

FIG. 5. Phase-averaged axial velocity measurements as a function of the axial coordinate at selected times.

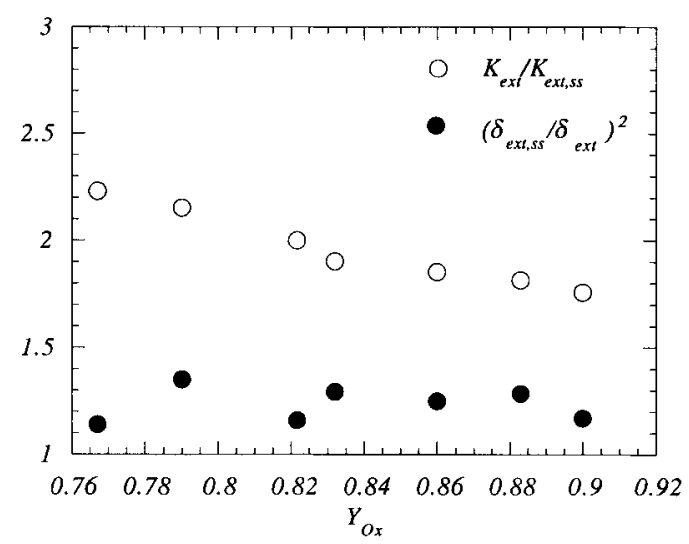

FIG. 6. Comparison of vortex-induced extinction strain rates and quasi-steady extinction values as a function of flame composition. The experimental values are normalized with the quasi-steady extinction strain rate. Also plotted are measurements of the square of the thickness of the mixing layer via HCHO PLIF, as estimates of normalized Damköhler number.

extinction curve by assuming a one-step reaction rate [18], with $\beta$ being the Zeldovich number, ranging between 8 and 12 for the present system; and the characteristic vortex turnover time, $\tau_{\text {vort }}=\pi(d / 2)^{2}$ / $\Gamma$, where is the circulation and $d$ is the vortex core diameter. The first two timescales can already be estimated at $\tau_{\mathrm{m}}=K^{-1}=17 \mathrm{~ms}$ and $\tau_{\mathrm{ch}} \approx 0.2-0.3$ $\mathrm{ms}$. The values of the vortex characteristic dimension and of the circulation for the case of $Y_{\mathrm{O}_{2}}=0.9$ were estimated at $d=1.2 \mathrm{~mm}$ and $\Gamma=5 \times 10^{-3} \mathrm{~m}^{2} / \mathrm{s}$, respectively. As a result, the vortex turnover time was estimated at $\tau_{\text {vort }}=2.4 \mathrm{~ms}$. The characteristic timescales, $\tau_{\mathrm{m}}=K^{-1}=17 \mathrm{~ms}, \tau_{\mathrm{ch}} \approx 0.2-0.3 \mathrm{~ms}$, and $\tau_{\text {vort }}=2.4 \mathrm{~ms}$, can now be ordered as $\tau_{\mathrm{ch}}<\tau_{\text {vort }}<$ $\tau_{\mathrm{m}}$. The same ordering applies in the case in which $Y_{\mathrm{O}_{2}}$ was varied, with $\tau_{\text {vort }}$ generally increasing some- what because of the lower circulation necessary to bring the flames to extinction.

To determine the vortex time, we first need to evaluate circulation and vortex core diameter from velocity measurements, as follows. A rectangular path was chosen for convenience, with one side along the centerline, two sides along the radial direction, and the last one parallel to the $z$ axis. The path was chosen in such a way to contain completely one-half of the toroidal vortex, and the minimal dimensions of this rectangle were established based on vortex visualization. After subtracting the steady velocity component, the temporal velocity measurements at the various locations were converted to a velocity field map at a fixed time, by following the same procedure illustrated above in connection with Fig. 5. Once this was done, the integral could be evaluated at any arbitrary time. For the circulation of the vortex to be meaningful, the integral needs to be calculated only when the vortex is fully enclosed in the integration path. The proper time at which the integral needed to be evaluated was chosen from vortex visualization. To estimate the other characteristics of the vortices, we followed the same approach as in Ref. [5]. Namely, the time traces of the axial component and of the radial component of the velocity were obtained at several locations along the radial direction for a fixed axial position. We phase averaged the data at the time at which the axial velocity was at a maximum in that specific axial position. Considering the toroidal vortex as a pair of counter-rotating vortices, we can localize the position of the vortex cores as the maxima of the radial velocity profile. The core dimension can be inferred from the axial velocity profile plot, as delimited by a relative maximum and a relative minimum. The vortex diameter can be defined as the distance between the two minima and the vortex translational velocity as the axial velocity at the core location.

A simple scaling argument, consistent with the characteristic time inequality, can now be used to explain the results of Fig. 6. Consider the mixing layer between two counterflowing jets of fuel and air with an overall chemical reaction that is strongly sensitive to the temperature. In the fast burning regime, the extinction of the diffusion flame occurs when, with increasing strain rates, the mass burning rate of fuel per unit flame surface increases above a critical value. This phenomenon can be described in terms of a characteristic Damköhler number that is based on the ratio of a mixing time over a chemical time. As a fluid time, we can use the inverse of the scalar dissipation rate at the stoichiometric surface, $1 / \chi_{\mathrm{s}}$. The scalar dissipation rate is defined in terms of the thermal diffusivity, $D_{\mathrm{T}}$, and of the square of the gradient of the mixture fraction evaluated at the stoichiometric surface, $|\nabla Z|_{s}^{2}$. The inverse of the mixture fraction gradient, $|\nabla Z|_{s}$, is a measure of the thickness of the mixing layer, $\delta$. Thus, 


$$
\chi_{\mathrm{s}}=D_{\mathrm{T}}|\nabla Z|_{\mathrm{s}}^{2} \approx \frac{D_{\mathrm{T}}}{\delta^{2}}
$$

If $\tau_{\mathrm{ch}}$ is the chemical time, the Damköhler number is defined as $D a=1 / \chi_{\mathrm{s}} \tau_{\mathrm{ch}}$. The approximate equality in equation 1 is necessary to account for a constant depending only on the value of the mixture fraction at the stoichiometric surface.

From the definition of $D a$, one can write the ratio of Damköhler number at extinction under vortex excitation, $D a_{e x t}$, to the same number under quasisteady extinction, $D a_{\text {ext,ss }}$, as

$$
\frac{D a_{\mathrm{ext}}}{D a_{\mathrm{ext}, \mathrm{ss}}}=\left[\frac{1}{\tau_{\mathrm{ch}}} \frac{\delta_{\mathrm{ext}}^{2}}{D_{\mathrm{T}}}\right]\left[\frac{D_{\mathrm{T}}}{\delta_{\mathrm{ext}, \mathrm{ss}}^{2}} \tau_{\mathrm{ch}}\right]=\left(\frac{\delta_{\mathrm{ext}}}{\delta_{\mathrm{ext}, \mathrm{ss}}}\right)^{2}
$$

since, in the first approximation, $\tau_{\mathrm{ch}}$ and $D_{T}$ are not affected by the perturbation.

If the strain rate, $K$, is changed with time, by either modulating the velocity in one of the feed streams or by means of a vortex generated near the axis of the jet, as in the present experiments, $\delta$ and, hence, $\chi_{\mathrm{s}}$, will also change with time. If $\chi_{\mathrm{s}}$ grows above the critical value required to extinguish the diffusion flame-that is, if $D a<D a_{\text {ext }}$-extinction occurs [18]. Using the peak extinction strain rate to characterize the counterflow diffusion flames is potentially misleading. The extinction behavior should be better characterized in terms of Damköhler number, scalar dissipation, or mixing layer thickness, which are the relevant quantities governing the flame burning rate up to extinction, as suggested by theory. The measurement of the HCHO layer thickness, with the justifications presented in connection with the discussion of Fig. 2, provides a convenient vehicle to achieve this goal, bypassing the need for detailed species measurements for the assessment of the scalar dissipation and its gradients.

The thickness of the mixing layer was measured at a strain rate within $5 \%$ of the extinction value. Small increments of strain rate caused the flame to extinguish. In the unsteady case, the thickness of the mixing layer was evaluated following the evolution in time of the formaldehyde layer and was estimated as the minimum value reached before extinction occurred. Error associated with this measurement is estimated to be on the order of $10 \%$. The square of the ratio of the quasi-steady to the unsteady extinction layer thickness, $\left(\delta_{\text {ext,ss }} / \delta_{\text {ext }}\right)^{2}$, that is the inverse of the right-hand side of equation 1, is shown in Fig. 6, as measured by HCHO PLIF. Differences of a factor larger than 2 between vortex-induced and quasi-steady extinction strain rates are now reduced to typically $25 \%$ in terms of scalar dissipation rate, or, equivalently, $D a$. This finding is consistent with the characteristic time inequality that suggests that no significant differences should be found between vortex-induced and quasi-steady extinction in terms of scalar dissipation rate or Damköhler number. Physically, the vortex introduces unsteady effects in the outer diffusive-convective layer. Specifically, the strain rate at the edge of the mixing layer increases, which results in a change of the thickness of the mixing layer, occurring on its own characteristic timescale. Extinction will occur when this thickness reaches a critical value that can be related to the scalar dissipation rate via equation 1 . The inner reactive-diffusive layer, on the other hand, behaves in a quasi-steady manner, since the characteristic chemical time is much smaller than the characteristic unsteady time. As a result, even though the instantaneous strain rate is much larger than the quasisteady extinction strain rate, the flame is subject to a damped strain rate through the outer layer. This procedure can be shown to be equivalent to determining an effective scalar dissipation rate that is affected by the time history of the strain rate [19].

We observe that implicit in the argument set forth is that the two-phase nature of the flow does not dramatically alter the flame structure. This assumption is likely to be valid in the present case, since in some respects these flames behaved as a pseudogaseous flame, with no significant evidence of direct droplet-flame interaction [20]. In other words, the droplets fully vaporized before reaching the flame, and none of them penetrated it. Another implicit assumption is that the vortex-flame interaction can be characterized essentially as an unsteady interaction, with the role of curvature being secondary. Comparison of the characteristic radius of curvature of the vortex and of the mixing layer thickness showed that the first is typically a factor of 5 larger, which is consistent with this assumption.

The explanation provided to rationalize the results in Fig. 6 in terms of Damköhler number should yield values scattered around the unity ordinate. The full symbols, on the other hand, are scattered around an ordinate mean value of 1.2. Other factors, in addition to experimental errors, may explain the inadequacy of the model. In this context, one should bear in mind that the simplified three-zone description of the flame structure, with an inner reactive-diffusive zone sandwiched by two outer convective-diffusive zones, is conceived within the framework of one-step kinetics and, consequently, of a single characteristic chemical time. For the purpose of this discussion, we can consider chemical time associated with the peak of heat release rate as the relevant one. In reality, there is a wide range of chemical times in a flame. For example, a significant fraction of the heat release is associated with the relatively slow $\mathrm{CO}$ oxidation. The simple three-zone model used here cannot capture these subtleties, but rather it emphasizes a physical description of the flame. Chemical kinetic issues must be addressed in necessarily computational studies, as, for example, in the insightful contribution in Ref. [21], where the chemical response 
TABLE 1

Stokes number estimates, based on the vortex turnover time, for different droplet sizes and gaseous temperatures

\begin{tabular}{|c|c|c|c|c|}
\hline \multirow[b]{2}{*}{$\begin{array}{l}d^{\prime} \\
(\mu \mathrm{m})\end{array}$} & \multicolumn{2}{|c|}{$T=300 \mathrm{~K}$} & \multicolumn{2}{|c|}{$T=600 \mathrm{~K}$} \\
\hline & St & $\begin{array}{c}\% \text { Mass for } \\
d<d^{\prime}\end{array}$ & St & $\begin{array}{c}\% \text { Mass for } \\
d<d^{\prime}\end{array}$ \\
\hline 15 & 0.18 & $1.3 \%$ & 0.07 & $8.6 \%$ \\
\hline 30 & 0.62 & $30 \%$ & 0.3 & $50 \%$ \\
\hline 40 & 1.12 & $60 \%$ & 0.6 & $84 \%$ \\
\hline 50 & 1.75 & $85 \%$ & 0.9 & $94 \%$ \\
\hline 60 & 2.4 & $95 \%$ & 1.2 & $98 \%$ \\
\hline
\end{tabular}

to unsteady perturbations of various pollutants is analyzed.

\section{Reignition Behavior and Droplet Inertia}

A noticeable difference was observed when spray flames were compared with gaseous ones with respect to the time between the onset of local extinction and the complete reconstitution of the flamethat is, when the extinction hole closed in. For example, for the flame with $Y_{\mathrm{O}_{2}}=0.9, \Delta t=26 \mathrm{~ms}$ for the spray flame, as compared with $\Delta t=11 \mathrm{~ms}$ for the same flame in which the liquid flow rate was deliberately prevaporized before exiting the burner mouth. A likely culprit is droplet inertia. The vortex, after penetrating the flame and entering the fuel side, centrifuges the droplets away from the centerline, reducing the amount of fuel supplied locally to the reaction zone and retarding the re-establishment of the appropriate edge flame propagation conditions, as compared with the gaseous flame analogue. Estimates of the relevant Stokes number confirmed this explanation and are reported in Table 1 . The Stokes number was calculated by comparing the particle stopping time, $\tau_{\mathrm{p}}=\left(\rho_{\mathrm{l}} d^{2}\right) /(18 \cdot \mu)$, with $\tau_{\text {vort }}$ [22]. In Table 1, selected Stokes numbers are calculated for two gas temperatures and different droplet sizes. For each size, the numbers in the third and fifth columns present the percentage of the total liquid mass in the distribution in droplets of a size smaller than the selected one. Regardless of temperature, these estimates show that inertia effects, that usually set in for $S t>0.1$, are significant for a sizable portion of the liquid mass, which gives credence to the proposed explanation for the delayed ignition.

\section{Conclusions}

Extinction strain rates of methanol counterflow spray diffusion flames were quantified using a com- bination of formaldehyde PLIF and phase Doppler measurements. Vortex-induced extinction resulted in values a factor of 2 larger than the quasi-steady extinction values. The difference was explained phenomenologically by estimating the timescales involved in the process. An estimate of the thickness of the mixing layer, based on formaldehyde PLIF, allowed for a comparison of the scalar dissipation rate and the Damköhler number between the two extinction modes. Such a comparison showed that the difference between the two modes was reduced to $25 \%$ on the average, consistent with expectations based on flame structure models from asymptotics. A comparison of the time delay between the onset of extinction and reignition between spray flames and gaseous flames showed that spray flames have much longer time delays. The difference was attributed to droplet inertia effects, as confirmed by estimates of the relevant Stokes number.

\section{Acknowledgments}

The authors wish to thank the following colleagues: Mr. Ernst Ebert, for the design and construction of the vortex generation system and its retrofitting to the counterflow burner; Mr. Mikhail Noskov, for preliminary calculations that enabled us to use HCHO as a flame marker; Dr. Adonios N. Karpetis, for helping with aspects of the data acquisition; and Professor Amable Linan, for enhancing our understanding of flame structure issues. The support of the National Aeronautics and Space Administration, under the Microgravity Science and Applications Program, grant no. NAG3-1688 (Mr. Dennis Stocker, contract monitor), and of the National Science Foundation, grant no. CTS9904296 (Dr. Farley Fisher, contract monitor), is gratefully acknowledged.

\section{REFERENCES}

1. Marble, F. E., in Recent Advances in the Aerospace Sciences (C. Casci, ed.), Plenum, New York, 1985, p. 395.

2. Ashurts, W. T., and Williams, F. A., Proc. Combust. Inst. 23:543-550 (1990).

3. Cuenot, B., and Poinsot, T., Proc. Combust. Inst. 25:1383-1390 (1994).

4. Roberts, W. L., and Driscoll, J. F., Combust. Flame 87:245-256 (1991).

5. Driscoll, J. F., Sutkus, D. J., Roberts, W. L., Post, M. E., and Goss, L. P., Combust. Sci. Technol. 96:213229 (1994).

6. Rolon, J. C., Aguerre, F., and Candel, S., Combust. Flame 100:422-429 (1995).

7. Thevenin, D., Renard, P. H., Rolon, J. C., and Candel, S., Proc. Combust. Inst. 26:1079-1086 (1996).

8. Thevenin, D., Rolon, J. C., Renard, P. H., Kendrick, 
D. W., Veynante, D., and Candel, S., Proc. Combust. Inst. 27:719-726 (1998).

9. Renard, P. H., Rolon, J. C., Thevenin, D., and Candel, S., Combust. Flame 117:189-205 (1999).

10. Katta, V. R., Carter, C. D., Fiechtner, G. J., Roquermore, W. M., Gord, J. R., and Rolon, J. C., Proc. Combust. Inst. 27:587-594 (1998).

11. Shiah, S. M., and Sichel, M., AIAA paper 93-0901, 1993.

12. Gao, L. P., D’Angelo, Y., Silverman, I., Gomez, A., and Smooke, M. D., Proc. Combust. Inst. 26:1723 (1997).

13. Massot, M., Kumar, M, Smooke, M. D., and Gomez, A., Proc. Combust. Inst. 27:1975-1983 (1997).

14. Santoro, V. S., Kyritsis, D. C., and Gomez, A., “A Comparison of the Interaction of Laminar Vortices with Either Gaseous or Spray Counterflow Diffusion Flames," Joint Meeting of the U.S. Sections of the Combustion Institute, Washington, DC, March 15-17, 1999.
15. Bauerle, B., Warnatz, J., and Behrendt, F., Proc. Combust. Inst. 26:2619-2626 (1996).

16. Paul, P. H., and Najim, H. N., Proc. Combust. Inst. 27:43-50 (1997).

17. Seshadri K, Trevino, C., and Smooke, M. D., Combust. Flame 76:111-132 (1989).

18. Liñán, A., Acta Astronaut. 1:1007-1039 (1974).

19. Howarth, D. C., Drake, M. C., Pope, S. B., and Blint, R. J., Proc. Combust. Inst. 22:589-597 (1988).

20. Gomez, A., and Chen, G., in Physical and Chemical Aspects of Combustion-A Tribute to Irvin Glassman (F. L. Dryer, and R. F. Sawyer, eds.), Gordon and Breach, Amsterdam, The Netherlands, 1997, p. 461.

21. Im, H. G., Chen, J. H., and Chen, J. Y., Combust. Flame 118:204-212 (1999).

22. Rosner, D. E., Transport Processes in Chemically Reacting Flow Systems, Butterworth-Heinemann, Stonehan, MA, 1986. 